\title{
Corrigendum: Gene-associated markers provide tools for tackling illegal fishing and false eco-certification
}

Einar E. Nielsen, Alessia Cariani, Eoin Mac Aoidh, Gregory E. Maes, Ilaria Milano, Rob Ogden, Martin Taylor, Jakob Hemmer-Hansen, Massimiliano Babbucci, Luca Bargelloni, Dorte Bekkevold, Eveline Diopere, Leonie Grenfell, Sarah Helyar, Morten T. Limborg, Jann T. Martinsohn, Ross McEwing, Frank Panitz, Tomaso Patarnello, Fausto Tinti, Jeroen K.J. Van Houdt, Filip A.M. Volckaert, Robin S. Waples, FishPopTrace consortium \& Gary R. Carvalho

Nature Communications 3:851 doi: 10.1038/ncomms1845 (2012); Published 22 May 2012; Updated 15 Jul 2013

In the Accession codes section and in Supplementary Table S2 of this Article, some of the herring SNP loci identification numbers and their associated NCBI dbSNP database accession codes are incorrect, as follows:

Cha 1025.1-149/ss503773365 should be Cha_10193.1-449/ss538786515

Cha 1170.1-250/ss503773374 should be Cha_1165.2-123/ss538786521

Cha 13197.4-115/ss503773376 should be Cha_13178.2-124/ss538786526

Cha 10733.1-102/ss503773379 should be Cha_1068.2-349/ss538786516

Cha $13371.3-81 /$ ss503773381 should be Cha_13259.1-167/ss538786527

Cha 11922.3-225/ss503773391 should be Cha_11896.1-201/ss538786522

Cha 12119.7-153/ss503773398 should be Cha_1203.1-312/ss538786523

Cha 1212.4-260/ss503773404 should be Cha_12119.7-664/ss538786524

Cha124.5-901/ss503773406 should be Cha_12381.1-143/ss538786525

Cha 14331.1-140/ss503773408 should be Cha_143.1-185/ss538786529

Cha 11251.1-463/ss503773416 should be Cha_11197.1-111/ss5387865186

Cha 13427.1-146/ss503773421 should be Cha_13376.1-166/ss538786528

Cha 117.1-661/ss503773426 should be Cha_1162.1-193/ss538786520

Cha $14998.1-247 /$ ss503773428 should be Cha_14942.1-236/ss538786530

Cha 11326.1-297/ss503773434 should be Cha_113.2-2374/ss538786519

Cha 11183.5-167/ss503773439 should be Cha_1104.1-937/ss538786517 